\title{
Juno Waves detection of dust impacts near Jupiter
}

Ye, S.Y.; Averkamp, T. F.; Kurth, W. S.; Brennan, M.; Bolton, S.; Connerney, J. E. P.; Jørgensen, J. L.

Published in:

Journal of Geophysical Research: Planets

Link to article, DOI:

10.1029/2019JE006367

Publication date:

2020

Document Version

Peer reviewed version

Link back to DTU Orbit

Citation (APA):

Ye, S. Y., Averkamp, T. F., Kurth, W. S., Brennan, M., Bolton, S., Connerney, J. E. P., \& Jørgensen, J. L. (2020). Juno Waves detection of dust impacts near Jupiter. Journal of Geophysical Research: Planets, 125(6). https://doi.org/10.1029/2019JE006367

\section{General rights}

Copyright and moral rights for the publications made accessible in the public portal are retained by the authors and/or other copyright owners and it is a condition of accessing publications that users recognise and abide by the legal requirements associated with these rights.

- Users may download and print one copy of any publication from the public portal for the purpose of private study or research.

- You may not further distribute the material or use it for any profit-making activity or commercial gain

- You may freely distribute the URL identifying the publication in the public portal

If you believe that this document breaches copyright please contact us providing details, and we will remove access to the work immediately and investigate your claim 
Ye Sheng-Yi (Orcid ID: 0000-0002-3064-1082)

Averkamp Terrance F. (Orcid ID: 0000-0001-6977-3472)

Kurth William S. (Orcid ID: 0000-0002-5471-6202)

Brennan Martin (Orcid ID: 0000-0003-0796-4251)

Bolton Scott J. (Orcid ID: 0000-0002-9115-0789)

Connerney John E. P. (Orcid ID: 0000-0001-7478-6462)

Joergensen John L. (Orcid ID: 0000-0002-0343-239X)

\section{Juno Waves detection of dust impacts near Jupiter}

S.-Y. Ye ${ }^{1 *}$ T. F. Averkamp ${ }^{1}$, W. S. Kurth ${ }^{1}$, M. Brennan'2, S. Bolton ${ }^{3}$, J. E. P.

\section{Connerney ${ }^{4,5}$, J. L. Joergensen ${ }^{6}$}

${ }^{1}$ Department of Physics and Astronomy, The University of Iowa, Iowa City, IA

${ }^{2}$ Jet Propulsion Laboratory, Pasadena, CA

${ }^{3}$ Southwest Research Institute, San Antonio, TX

${ }^{4}$ NASA Goddard Space Flight Center, Greenbelt, MD

${ }^{5}$ Space Research Corporation, Annapolis, MD

${ }^{6}$ Technical University of Denmark, Kgs. Lyngby, Denmark

*Current address: Department of Earth and Space Sciences, Southern University of Science and Technology (SUSTech), Shenzhen, China

Corresponding author: W. S. Kurth, william-kurth@uiowa.edu

This article has been accepted for publication and undergone full peer review but has not been through the copyediting, typesetting, pagination and proofreading process which may lead to differences between this version and the Version of Record. Please cite this article as doi: 10.1029/2019JE006367 


\section{Key points}

- The Juno Waves instrument detected voltage pulses induced by dust impacts

- Impact rates peak around the ring plane with half width at half max $\sim 2000-3000 \mathrm{~km}$

- Most of the impacts detected are on the spacecraft and not the Waves antenna

\section{Abstract}

The Juno spacecraft entered orbit at Jupiter on July 5, 2016. Since then, Juno has orbited Jupiter in high inclination orbits, crossing the ring plane near perijove. During 20 of the first

21 crossings, the Waves instrument detected signals associated with dust impacts. The impact rate profiles show peaks of order $6 \mathrm{~s}^{-1}$ around the ring plane with half width at half maximum 2000-3000 km. The polarity ratio of the impact signals didn't follow the areas of the antennas exposed to dust impacts that change due to the rotation of the spacecraft, suggesting Waves detects impacts on the Juno spacecraft and not just on the Waves antennas. The impact rate profile changed during Perijove 19, when the spacecraft rotation axis was tilted to the south, increasing the area of the solar panels exposed to impacts, indicating that the detected impacts were on the spacecraft body. Grain sizes of order $1 \mu \mathrm{m}$ are estimated, and the differential size distribution has a slope of -5.1 and with number densities of order $3 \times 10^{-}$ ${ }^{6} \mathrm{~m}^{-3}$

\section{Plain Language Summary}

Once each orbit, Juno passes within a few to several thousand kilometers of Jupiter's cloudtops at low latitudes. These passes carry the spacecraft through Jupiter's ring plane. The radio and plasma wave instrument on Juno called Waves detects voltage spikes when passing through this plane, even though Juno is well below what is thought to be the inner edge of the known ring system. These spikes are due to impacts by micron-sized grains of 
dust at relative speeds upwards of $70 \mathrm{~km} / \mathrm{s}$. At such speeds, the dust and even a tiny part of the spacecraft is immediately vaporized and heated to a high enough temperature to form a rapidly-expanding cloud of electrons and ions. These clouds momentarily disrupt the potential around the spacecraft causing an impulse in the Waves electric sensor. During the first 21 Juno orbits we find peak impact rates of about 6 per second mainly confined to within $2000-3000 \mathrm{~km}$ of the ring plane. We estimate the size of these grains in the range of microns, about the size of particles in cigarette smoke.

\section{Introduction}

This paper uses observations from the first 21 polar orbits since Juno's July 5, 2016 arrival at Jupiter. The highly elliptical orbit has a period of 53 days with the spacecraft spending a few hours close to the planet near each perijove. The location of the perijoves precesses about $1^{\circ}$ north each orbit, beginning very close to the equator at orbit insertion, but stays within 3500$8000 \mathrm{~km}$ above the cloud tops (Bolton et al., 2017). During each of the perijoves for which science data were collected, voltage signals due to dust impacts were detected by the dipole antenna of the Waves instrument (Kurth et al., 2017), providing the first in-situ measurement of dust particles in the region between Jupiter and its ring.

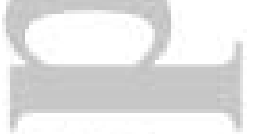

Jupiter's main ring was first imaged by Voyager 1 cameras (Smith et al., 1979), targeting the equator at $1.8 \mathrm{R}_{\mathrm{J}}$ radial distance, where a particulate ring was thought to exist, absorbing energetic electrons (Acuña and Ness, 1976; Fillius, 1976). Jupiter's ring consists of four main components: the main ring (optical depth $\tau \sim$ a few $10^{-6}$ ) between the orbits of Metis and Adrastea $\left(1.72\right.$ to $1.81 \mathrm{R}_{\mathrm{J}}$, where $\left.\mathrm{R}_{\mathrm{J}}=71,492 \mathrm{~km}\right)$, the torus-shaped halo ring $\left(\tau \sim 10^{-6}\right)(1.4$ $1.72 \mathrm{R}_{\mathrm{J}}$ ), and the two faint gossamer rings $\left(\tau \sim 10^{-7}\right)$ roughly coinciding with the orbits of Amalthea and Thebe (1.81-3.2 RJ) (Burns et al., 1984; Showalter et al., 1985, 1987; Ockert- 
Bell et al., 1999). For comparison, Juno crossed the ring plane between 1.04 and $1.1 \mathrm{R}_{\mathrm{J}}$. The grains in the ring are predominantly micron-sized and believed to be generated by micrometeorite impacts on and collisions between parent bodies (moons and large particles) in the ring (Burns et al., 1999; de Pater et al., 2008). The main ring has a relatively larger fraction of macroscopic material (backscattering more light) compared to the fainter halo and gossamer rings. A fit of the phase curve based on a power law size distribution indicated a power law index of $\sim-2$ (Showalter et al., 2008).

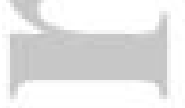

In this paper, we analyze the dust impact signals detected by Juno Waves. First, we briefly discuss the generation mechanism of the impact signals. Then we try to answer the question whether Juno detects only impacts on the Waves dipole antennas or on other parts of the spacecraft. Knowing the effective collecting area is crucial to understanding the number density of grains near Jupiter's equator between Jupiter and the ring. We present impact rate profiles measured during the perijoves and the particle mass distribution slope. These values provide important baseline checks for numerical simulations and the parameters derived from remote sensing.

We mention at the outset that the Advanced Stellar Compass (ASC) that is part of the Juno Magnetometer investigation (Connerney et al., 2017) has demonstrated the ability to detect impacts of grains larger than $1 \mu \mathrm{m}$ (Benn et al., 2017). The ASC, using the full size of the Juno S/C, can detect dust particles larger than $\sim 1 \mu \mathrm{m}$ impacting the back side of the solar panels with a detection efficiency of $\sim 7 \%$. ASC particle detection was enabled throughout approach, orbit insertion, and in orbit until orbit 7, and found that the detection rate dropped from a very high count rate on approach, to zero after orbit insertion. This result is to be expected if existing grains are in prograde Keplerian orbits as these would impact the sun- 
facing side of the panels and not be observable by the ASC. After orbit 7, Jupiter is in the field of view of ASC near perijove, and as a result, dust detection near the equator with ASC is not possible until much later in the mission. Hence, we will not further discuss the ASC observations.

\section{Detection mechanism}

Electric field antennas onboard spacecraft were found to be sensitive to dust impacts during the Saturn flybys by the Voyagers (Gurnett et al., 1983; Aubier et al., 1983), and since then has provided important in-situ observations of dust particles in space (Gurnett et al., 1987; Gurnett et al., 1991; Pedersen et al., 1991; Meyer-Vernet et al., 1996; Tsurutani et al., 2004; Wang et al., 2006; Kurth et al., 2006; Meyer-Vernet et al., 2009; Zaslavsky et al., 2012; Ye et al., 2014b; Malaspina et al., 2014; Kellogg et al., 2016; Vaverka et al., 2017; Vaverka et al., 2019). Models have been proposed to interpret the signals observed (Meyer-Vernet 1985; Oberc, 1996; Zaslavsky, 2015; Meyer-Vernet et al., 2017), emphasizing the role of charge recollection in creating a voltage difference between the spacecraft and antennas.

Recent analyses based on Cassini observations, laboratory experiments, and numerical simulations clarified that the different movements of the electrons and ions in the impact plasma cloud are responsible for generating the potential perturbation (Ye et al., 2019). The electrons and ions of the impact plasma cloud have about the same temperature due to thermalization during cloud expansion (Collette et al. 2016), meaning electrons escape much faster than ions once the gas is beyond its collisional stage due to their smaller mass. The resulting charge separation changes the potential of the impact target (Meyer-Vernet et al., 1986; Oberc et al., 1990; Pantellini et al., 2012). The particle trajectories can be influenced by the spacecraft potential thereby affecting the escape of particles. It is shown in laboratory 
simulations that the polarity and time scale of the initial part of the impact signal depends on whether the potential perturbation is caused by electron or ion escape (Nouzák et al., 2018; Ye et al., 2019).

In the present work, the Juno Waves electric antenna is used to detect dust impacts. This antenna is operated as a dipole antenna in which the voltage difference between the two elements is detected. However, electric field antennas can also be operated in a monopole mode (although this is not possible for Juno Waves) in which the potential on each element is referenced to the spacecraft chassis potential. The monopole configuration is more sensitive to dust impacts than the dipole configuration (Meyer-Vernet, 1985, Tsintikidis et al., 1994; Meyer-Vernet et al., 2014). A dipole antenna is often used to provide common-mode rejection, reducing interference from noise on the chassis. But, this also results in a lower sensitivity to dust impacts on a spacecraft body that primarily modify the chassis potential. Laboratory simulations have been used to increase understanding of impact signals (Collette et al., 2015). These have investigated the differences in responses by monopole versus dipole antennas (Nouzák et al., 2018). This work showed that in the dipole mode the antennas are relatively insensitive to dust impacts occurring on the spacecraft and only impacts on the antennas generate clear signals. Ye et al. (2016a) showed different impact rates detected by the monopole and dipole antenna configurations when Cassini RPWS switched antenna modes during ring plane crossings. The differences reflect different effective collecting areas to be used for calculating the density of dust encountered by the spacecraft.

\section{Observations}

Figure 1 shows Juno Waves observations of dust impacts during PJ21 at three different timescales. In Figure 1a, showing a frequency-time spectrogram of the Waves electric field 
data, the broadband bursty signals around the closest approach are due to dust impacts. Figure $1 \mathrm{~b}$ shows a 123 ms waveform capture which typically occurs once per second at a sample rate of $50 \mathrm{ksps}$ showing three individual impacts identified by an algorithm based on a minimum slope threshold in the voltage waveform and highlighted by the colored symbols (red for positive-going and green for negative going impulses). The first event was not flagged by the detection algorithm because the initial slope did not satisfy the criterion. Figure 1c shows the voltage waveform of an individual impact signal. The impact signal is characterized as a sudden increase in voltage followed by a slower recovery to equilibrium potential, often overshooting to the other polarity. These overshoots are due to the receiver response to a fastchanging signal and will decrease when applying the inverse-transfer function to the waveforms (Ye et al., 2019). The polarity of the signal sheds light on the location of the impacts (Malaspina et al., 2014; Ye et al., 2016b).

Figure 2 shows the impact rate profile for perijove 21. The rates are smoothed using a 25second sliding boxcar average. This profile is typical of the impact rates for most of the perijoves to this point in the mission and show a peak rate of about 6 impacts per second centered near 04:12. The quantization observed at low count rates is due to a combination of the limited duty cycle of the waveform observations ( $123 \mathrm{~ms}$ each second) and the 25 second averaging intervals. During this perijove, there appears to be a secondary peak near 04:14. This may suggest a secondary population of grains on slightly inclined orbits or simply low counting statistics. Suggestions of such a structure is sometimes seen in other orbits. We caution that the 30 -s rotation period of the spacecraft (hence impact geometry) is similar to the time it takes to pass through the densest region of the dust, and therefore, aliasing effects cannot be ruled out. 
Juno Waves employs a dipole antenna made of two antenna booms attached to the spacecraft body symmetrically deployed behind the solar panel array that has the magnetometer installed at the end (Kurth et al., 2017). The antenna elements, separated by $120^{\circ}$, are both 2.8 $\mathrm{m}$ long with three tapered sections with diameters that decrease toward the tip of the antenna booms giving an average diameter of about $0.95 \mathrm{~cm}$. During most perijoves, the spacecraft rotation axis is approximately parallel to the ring plane with the solar panels and high gain antenna facing the Earth for gravity measurements. During each 30 s rotation of the spacecraft, the solar panels occasionally block the electric antennas from impacting dust in Keplerian orbit about Jupiter, providing an opportunity to test whether dipole antennas are only sensitive to impacts on the antenna booms. Ye et al. (2016a) showed that the polarity ratio of the impact signals detected by the dipole antenna follows the projected antenna area ratio of the two antenna elements as Cassini rotated during an Enceladus flyby, which indicated that the instrument is primarily sensitive to impacts on the antenna elements when operated in the dipole mode. Figure 3 shows the count rates of positive and negative impact signals detected by Juno Waves during PJ20 compared with the exposed areas of antenna elements (in percentage), which vary periodically and sometimes reach zero due to the rotation of Juno and the shielding of antenna elements by the solar panels. Here, we assumed circular Keplerian orbits for the grains. The exposed areas of the Waves antenna elements were calculated using two independent methods, one geometrical and the other counting the pixels of exposed parts of antennas in a 3D spacecraft visualization program, which showed agreement between them. The positive and negative impact rates do not follow the variation of the exposed antenna areas, and impacts were detected even when the antenna elements are completely blocked by the solar panels, suggesting that the Waves instrument is also sensitive to impacts on the spacecraft body and solar panels when using the dipole antenna. This is discussed further in the Summary and Discussion section. 
Figure 4 depicts another way of investigating whether spacecraft attitude affects the rate of positive or negative impulses that might indicate a predominance of impacts on the Waves antenna elements. The three panels show the rate of positive, negative, and all impulses (top to bottom) as a function of spacecraft spin phase, where spin phase is arbitrarily measured from when the spacecraft $\mathrm{x}$ axis is parallel to Jupiter's equatorial plane. Here, we have summed all the impacts for perijoves 14, 19, and 20 to increase the counting statistics. Given the time it takes Juno to cross the peak in counting rates is only about a minute (see Figure 2) and the peak counting rate is only a few impacts per second, a single crossing does not provide sufficient statistics or phase coverage to reveal a significant result. Figure 4 shows no significant variation in impact rates of either polarity with spacecraft orientation, hence we, again, conclude that the impacts are primarily on the spacecraft and solar panels as opposed to only on the Waves antennas.

As mentioned above, the dipole antenna should be more sensitive to impacts on one of the antenna elements than on the spacecraft, so it is likely Juno Waves would also detect impacts on its antennas. The maximum cross-sectional area of the two Waves antenna elements is only $0.053 \mathrm{~m}^{2}$. The solar panels have a maximum area of order $60 \mathrm{~m}^{2}$ and the spacecraft, itself approximately $5 \mathrm{~m}^{2}$. Given the relative velocity of the dust in Keplerian orbit and the spacecraft attitude near the equator (which varies from orbit-to-orbit), the total cross-sectional area varies from about 40 to $20 \mathrm{~m}^{2}$. Hence, the probability of an impact on an antenna element is one in 800 to one in 400 smaller than on the spacecraft. In fact, we have recorded 66 impacts during perijoves $11-14,19$, and 20 at times when neither Waves antenna was exposed to impacts assuming a circular Keplerian dust orbit. The amount of time both antennas are simultaneously shielded from dust exposure is a small percentage, 46 waveform 
captures versus a total of 920 captures for these six perijoves. The total number of impacts detected for any level of antenna exposure was 1,317 . If one scales the impact rate during the 46 captures when both antennas were shielded to the full 920 captures, one might expect 1320 impacts. Hence, virtually all of the impacts detected could have occurred on the spacecraft. In fact, some impacts probably did occur on one or the other of the Waves antennas, but these probably account for a fraction of one percent of all impacts or less, simply based on the ratio of the cross-sectional area.

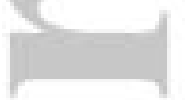

Figure 5 shows the impact rates detected by Juno Waves during perijoves $1-21$. No data were collected during perijove 2 . The impact rate vs. $\mathrm{Z}$ (elevation from the ring plane) profiles show peaks near the ring plane of around 6 impacts/sec with a half-width at halfmaximum (HWHM) of around 2000-3000 km. Table 1 lists Lorentzian function fit parameters of the impact rate profiles PJ 1-21. From Figure 5 and Table 1, we noticed that the impact rate profile peak of PJ19 is significantly wider than that of the other perijoves, which are relatively consistent with each other. The difference between PJ19 and other perijoves is that the rotation axis of Juno was tilted south with respect to the ring plane so that the projected area of the solar panels in the ram direction was increased by $\sim 30 \%$. This would have increased Juno's sensitivity to dust impacts as the effective impact area was increased. However, why the peak impact rate near the ring plane crossing remained at $\sim 6$ impacts/sec is puzzling.

The fact that most of the impacts detected by Waves likely occurred on the solar panels or the spacecraft body is important as this sets the effective collecting area to something on the order of 20 to $40 \mathrm{~m}^{2}$ as opposed to $0.053 \mathrm{~m}^{2}$. The variation is due to the evolving crosssection facing dust in Keplerian orbit near normal to the plane of the solar panels near dusk 
(close to PJ1) to more parallel to the plane of the solar panels near local noon (close to PJ22) in each case. In addition, the spin axis attitude is sometimes adjusted from its nominal Earthfacing attitude (for gravity measurements) to maximize viewing by the Microwave Radiometer investigation. Given a maximum impact rate of $\sim 6 \mathrm{~s}^{-1}$ (see below), the inferred number density of grains is 400 to 800 times smaller if the majority of detected impulses are on the spacecraft and not the Waves antennas. The number density $d$ of grains in the region is related to the impact rate $r$, the effective collecting area $A_{\text {eff }}$, and the relative velocity between the dust and spacecraft $v$ by $\quad r=d A_{\text {eff }} v$. Taking $v=60 \mathrm{~km} / \mathrm{s}$ and $A_{\text {eff }}=30 \mathrm{~m}^{2}, d$ $=3.3 \times 10^{-6} \mathrm{~m}^{-3}$. This estimate does not take into account the possibility of varying charge yields for impacts on different surface materials on the spacecraft or the possible effect of diminishing impulse amplitudes from impacts at large distances from the Waves antenna, e.g. near the end of the solar panels.

Figure 6 shows the voltage size distribution of the impact signals detected during the first 21 perijoves. The Waves instrument incorporates fixed attenuation levels that are used based on the onboard amplitude detection. Before a switch to a greater level of attenuation, the dust impact pulses may be clipped. Hence, the population of impact signals used in the distribution in Figure 6 includes only non-clipped impulses, but at any attenuation level in effect at the time of the impulse. Of course, the amplitude is calibrated with the attenuation then in effect. Since the voltage pulse height is proportional to the charge released during the dust impact, which is proportional to the mass of the impacting particle, the mass of the particles encountered can, in principle, be inferred from the voltage, given the material of the particle and the impacted surface (Collette et al., 2014). Given impacts on the spacecraft detected by the dipole antenna and not on the antenna elements, themselves, an accurate determination of the mass (or radius) of the grains is problematic. On the other hand, the 
slope of the size distribution should be reasonably reliable. The power law function fit of the differential mass distribution shows a slope around -1.7 , which is equivalent to a size distribution slope of -5.1 , similar to the -5 size slope predicted by the simulation of Horanyi and Juhasz (2010). We also determined the size distribution using just the lowest gain state (attenuation on) and found a voltage pulse height distribution with a slope of -1.3 or a size distribution with slope of -3.9. Use of just those events detected with the attenuator on limits the number of events and, also favors larger impacts. Hence, we prefer to rely on the distribution using both attenuator settings.

An in-depth discussion of the ramifications of the observed size distribution is beyond the scope of this study. However, the relatively steep distribution indicates a predominance of smaller particles and few larger grains that would contribute to the optical depth. The smaller particles are most influenced by electromagnetic and other non-gravitational forces. It is also the case that the observed distribution is almost certainly evolved from the parent distribution through differential losses as a function of size and position. Models of these smaller grains, like those of Horányi and Juhász (2010) can be used to estimate the lifetime of the population.

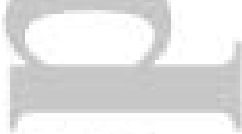

As mentioned above, the determination of grain mass from the Juno Waves observations is problematic, given a range of possible target materials and locations. However, we provide the following order-of-magnitude estimate of the grain masses making a number of assumptions, some of which are likely to be poor, at the outset. We start with a scaling argument used by Kurth et al. (2006) that used an estimate of root mean square grain masses from Cassini's observations near Saturn orbit insertion (SOI) between the F and G rings of $7.7 \times 10^{-11} \mathrm{~g}$ (Wang et al., 2006). The basis for the scaling is the voltage produced by 
impacts scales as $K m v^{\beta}$ where $K$ is a yield constant, $m$ is the particle mass, $v$ is the relative speed of the impact and $\beta$ is an empirically-determined constant. We use $\beta=3.5$ after Schippers et al. (2015). $K$ is a function of target and grain composition and incorporates the efficiency of coupling of the charge cloud produced by the impact to the antenna. For lack of a detailed understanding of $K$, we assume for this approximation that this yield constant is the same order of magnitude for both Cassini at Saturn and Juno near Jupiter. Then, we can say:

$$
\frac{V_{\text {Cas }}}{V_{\text {Jno }}}=\frac{m_{\text {Sat }}}{m_{\text {Jup }}}\left(\frac{v_{\text {Sat }}}{v_{\text {Jup }}}\right)^{\beta}
$$

Where $V_{\text {Cas }}$ and $V_{\text {Jno }}$ are the rms voltages observed by Cassini and Juno, respectively; $m_{\text {Sat }}$ and $m_{\mathrm{Jup}}$ are the rms grain masses at Saturn and Jupiter, respectively, and $v_{\mathrm{Sat}}$ and $v_{\mathrm{Jup}}$ are the impact speeds encountered by Cassini at Saturn and Juno at Jupiter, respectively. Assuming the $K \mathrm{~s}$ are the same means they do not enter the above equation.

The relative velocity for the Cassini impacts near SOI $v_{\text {Sat }}$ was $\sim 16 \mathrm{~km} / \mathrm{s}$ whereas for Juno the relative velocity is about $73 \mathrm{~km} / \mathrm{s}$ for perijove 11 and similar for others. Wang et al. (2006) found $V_{\text {rms }}$ during the SOI crossings to be $6.65 \mathrm{mV}$, which we use for $V_{\text {Cas }}$. In Figure 7 we show the mass distribution of particles $\mathrm{V}^{2} \mathrm{~F}(V)$ used in Figure 6. This gives $V_{\mathrm{Jno}}=0.649 \mathrm{~V}$. Substituting these values into the equation and solving for $m_{\text {Jup }}$ we obtain $m_{\text {Jup }} \sim 3.7 \times 10^{-11} \mathrm{~g}$. This is the basis for the mass scale at the top of Figure 6. Assuming silicates with a mass density $\rho_{\mathrm{m}} \sim 3 \mathrm{~g} / \mathrm{cm}^{3}$, the characteristic radius of the grains is about $1.4 \mu \mathrm{m}$, not unlike the sizes given by Burns et al. (1999) and de Pater et al. (2008). This is the basis for the selected grain sizes shown in Figure 6. Hence, to first order, the Jovian grains have masses similar to

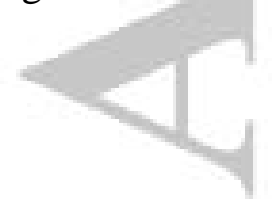


those between the F and G rings at Saturn. We repeat, however, that several assumptions have been made in arriving at this mass, so due caution should be used.

It is important to mention that the detection algorithm used to identify impacts uses a relatively large value of $\mathrm{dV} / \mathrm{dt}$. When we tried lower thresholds, we found increasing falsepositive detections based primarily on plasma waves also present in the near-Jupiter ring plane, such as whistler-mode waves. Given the high relative velocities of the Juno impacts, it is reasonable to expect Juno Waves would be sensitive to smaller grains. However, our study is limited to rather large $\mathrm{dV} / \mathrm{dt}$ values simply to avoid false-positive detections. Hence, the size distribution almost certainly extends to smaller sizes than shown in Figure 6, but we are reluctant to risk false detections inherent in extending to smaller grains.

\section{Summary and Discussion}

We have shown the Juno Waves detection of dust impacts in the region between Jupiter and its rings. While the stated inner edge of the halo ring is $1.4 \mathrm{R}_{\mathrm{J}}$, it is expected that some material can be ejected from the ring inwards of the 2:1 Lorentz resonance (Burns et al., 2004). As discussed in Horányi and Juhász (2010) the inward migration of dust is governed by gravity and electromagnetic forces, varying with grain size. Therefore, the detection of a small population of grains in this region is not entirely unexpected. The size distribution of the dust grains is proportional to $\mathrm{r}^{-5.1}$ with an estimated size of order $1 \mu \mathrm{m}$. The impact rates peaked at around 6-7 impacts/sec near the ring plane crossing with an HWHM around 2000$3000 \mathrm{~km}$. The variation of the impact rates separated by polarity does not follow the variation of the exposed areas of the antenna elements. There were impacts detected by the dipole antenna even when both antenna booms were blocked by the solar panels from dust particles in the ring plane. The impact rate vs. Z profiles are generally consistent for all perijoves so 
far except PJ19 (wider peak), during which Juno made a special maneuver to turn its solar panels more toward the south. This indicates that the Waves instrument is sensitive to impacts on the spacecraft body and the solar panels, while Ye et al. (2016b) showed that the Cassini dipole antennas were primarily sensitive to impacts on the antenna booms. It is almost certain Waves is sensitive to impacts on its antenna elements in addition to other parts of the spacecraft, but these likely comprise a small percentage of the impacts detected, given the large disparity in the spacecraft vs. antenna element cross-section. This is important in determining the number density of grains in the region which we estimate is $3.3 \times 10^{-6} \mathrm{~m}^{-3}$ taking $v=60 \mathrm{~km} / \mathrm{s}$ and $A_{\text {eff }}=30 \mathrm{~m}^{2}$.

Figure 8 shows densities assuming the spacecraft and solar panels comprise the effective area. We've estimated the effective area by using the maximum area multiplied by the absolute value of the sine of the angle between the incoming grains and the spacecraft $\mathrm{z}$-axis (high gain antenna axis) at the equator and used the relative speed between Juno and grains in circular Keplerian orbits for the velocity.

Horányi and Juhász (2010) made predictions about the dust that might be measured by Juno in its orbit taking it through the ring plane close to Jupiter. Figure 7 of Horányi and Juhász (2010) show somewhat smaller, but not dissimilar HWHM's to those we report, especially for smaller grains. In fact, the wings of their $0.4<a_{\mu}<1$ distribution, while smaller, are suggestive of the spread seen in the right-hand panel of Figure 8. Our ability to accurately determine the actual size of the detected grains is highly limited, hence, it could be that Juno is actually detecting smaller grains than the analysis in Figure 6 suggests.

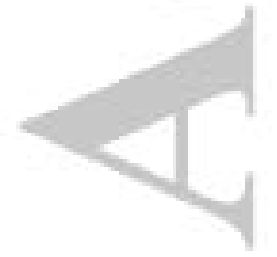


Horányi and Juhász (2010) predict expected fluences to be seen by Juno in a typical crossing through the equatorial region. We can estimate the fluence observed by Juno from Figure 2, taking the time the impact rates were at or above $3 \mathrm{~s}^{-1}$, which is about 13 minutes. Assuming an impact rate of $3 \mathrm{~s}^{-1}$ for $13 \times 60 \mathrm{~s}$ gives a conservative value of 2,340 impacts. Using the estimated exposed area of $25 \mathrm{~m}^{2}$ for PJ21, the fluence is $94 \mathrm{~m}^{-2}$. Based on their Figure 8, this falls between the expected fluence for $1-5 \mu \mathrm{m}$ and $5-10 \mu \mathrm{m}$ grains. Or, one could say that if Juno is truly detecting 1-2 $\mu \mathrm{m}$ grains as we determined in Figure 6, our fluence is a factor of 3 to 5 below the prediction. Overall, given our assumptions and large uncertainties, these results are largely in line with the predictions of Horányi and Juhász (2010).

Laboratory simulations of the antenna signals induced by dust impacts showed that the antennas are primarily sensitive to impacts on respective antenna units (dipole antenna sensitive to impacts on the dipole antenna booms and monopole antenna sensitive to impacts on the spacecraft body and the monopole antenna element) (Nouzák et al., 2018).

Occasionally, smaller signals were detected in the dipole channel even though the dust impacted on the spacecraft body or Ew (monopole) antenna. This was considered to be 'cross-talk' between the two preamplifiers in the spacecraft model, but the 'cross-talk' seems to occur only for high impact velocities, which were recorded by the instruments. The higher impact speed generates more impact charges, which would need to expand farther away from the spacecraft for its influence on the spacecraft potential to diminish. The ions and electrons in the expanding plasma cloud form two separate fronts which would perturb the potential of the dipole antenna elements in their path. The plasma cloud generated by a slower particle impact would decrease to the ambient plasma level before the escaping charges reach the antenna elements, thus would not induce any noticeable potential variation. This could explain the difference between the Juno and Cassini observations, as the impact speed for 
Juno near perijove was around $73 \mathrm{~km} / \mathrm{sec}$ while the impact speed for Cassini near Enceladus was only around $8 \mathrm{~km} / \mathrm{sec}$. This also suggests that the effective collecting area could be smaller for smaller grains, as a lower total charge yield would be less detectable if the impact were far from the Waves antenna. Hence, using a fixed collecting area could result in underestimating the number of smaller grains. Another difference could be the relatively short length of Juno's antennas with respect to the spacecraft (solar panels); Cassini's antennas were of the same order of scale as the Cassini spacecraft, or longer. Hence, the Juno Waves antenna is more likely embedded in the spacecraft sheath than was the case for Cassini; a detailed model of how the potential in the spacecraft sheath would affect the detectability of the plasma cloud may be needed.

The magnetic field strength at Jupiter's equator ranges from about 225 to $780 \mu \mathrm{T}$ based on Juno magnetometer (Connerney et al., 2017) measurements from the first 21 perijoves, compared to the $21 \mu \mathrm{T}$ magnetic field at the equator of Saturn (Belenkaya et al., 2006). Nouzák et al. (2019) examined the effect of ambient magnetic field, which controls the motion of electrons/ions in the impact plasma cloud, on the dust impact signal. The laboratory simulation showed that a stronger magnetic field reduces the Larmor radius of escaping electrons so that they are more likely to be recollected by the spacecraft, resulting in the reduction of the electron signals. Indeed, the electron Larmor radius in the vicinity of Jupiter (a few cm for a $10 \mathrm{eV}$ plasma cloud) is tiny compared to the scale of the spacecraft. The path of ions is not affected as much by the magnetic field, and for heavier elements the Larmor radii can be similar to the scale of Juno, depending on charge state. It was also pointed out that the higher ambient plasma density in Saturn's ionosphere played an important role in reducing the magnitude of the impact signals. At Jupiter, the peak impact rates do not change very much with perijove altitudes over the first 21 orbits. We have not 
completed an analysis of ionospheric densities along Juno's trajectory where grain impacts are detected, nor have we attempted to correlate impact rates with magnetic field intensity. Hence, it is premature to comment on the effects of the strong magnetic field or the plasma density on the dust impact signals at Jupiter.

Examples of dust impact signals detected by Juno Waves near Jupiter given in Figures 9 and 10 often have shapes more complicated than those observed by Cassini RPWS at Saturn (see examples in Figure 1 of Ye et al., 2019). More specifically, there are often multiple overshoots in an impact signal which can't be simply explained by the filter transfer function effect. The rise and decay parts of the Juno dust impact signals are not as smooth as the Cassini dust signals but are often characterized by multiple peaks and sometimes periodic oscillations. It is likely that the composition of dust particles in Jupiter's rings is more complicated than the water ice at Saturn, which upon impact would release multiple ions species in the impact plasma cloud. Higher velocity impacts at negative spacecraft potential would also yield negative ions and secondary electron emissions (Lee et al., 2013). The different charge carriers would expand at different velocities, generating multiple fronts in the expanding plasma cloud (Lee et al., 2012). The oscillations in the impact signals could be the low frequency part of the electromagnetic pulse generated by the oscillating ambipolar electric field during the plasma cloud expansion (Close et al., 2013). The escaping electrons could also induce plasma oscillations at ambient plasma frequency through the bump-on-tail instability (Ye et al., 2014a).

\section{Acknowledgements}

We thank S. M. Brooks for useful comments on this work. The research at the University of Iowa was supported by NASA through contract 699041X with Southwest Research Institute. 
The data used herein are archived in the JNO-E/J/SS-WAV-3-CDR-BSTFULL-V1.0 dataset in NASA's Planetary Data System at https://pds.nasa.gov. Specific higher level data discussed in this paper are archived in Zenodo (Ye et al, 2020).

\section{References}

Acuna, M. H., \& Ness, N. F. (1976). The main magnetic field of Jupiter. J. Geophys. Res., $81,2917-2922$.

Aubier, M. G., Meyer-Vernet, N., \& Pedersen B. M. (1983). Shot noise from grain and particle impacts in Saturn's ring plane. Geophys. Res. Lett., 10, 5-8.

https://doi.org/10.1029/GL010i001p00005

Belenkaya, E. S., Alexeev, I. I., Kalagaev, V. V., \& Blohhina, M. S. (2006). Definition of Saturn's magnetospheric model parameters for the Pioneer 11 flyby. Annales Geophysicae, 24 (3), 1145-56. https://doi.org/10.5194/angeo-24-1145-2006

Benn, M., Jørgensen, J. L., Denver, T., Brauer, P., Jørgensen, P. S., Andersen, A. C., ... Levin, S. M. (2017). Observations of interplanetary dust by the Juno magnetometer investigation. Geophysical Research Letters, 44(10), 4701-4708.

https://doi.org/10.1002/2017GL073186

Bolton, S. J., Lunine, J., Stevenson, D., Connerney, J. E. P., Levin, S., Owen, T. C., et al. (2017). The Juno mission. Space Science Reviews, 213(1-4), 5-37.

https://doi.org/10.1007/s11214-017-0429-6 
Burns, J. A., Schaffer, L. E., Greenberg, R. J., \& Showalter, M. R. (1984). Numerology or

Not? Jovian Ring Features are Near Resonances. Bulletin of the American Astronomical Society, 16, 675.

Burns, J. A., Showalter, M. R., Hamilton, D. P., Nicholson, P. D., de Pater, I., Ockert-Bell, M. E. \& Thomas, P. C., 1999. The formation of Jupiter's faint rings. Science, 284(5417), 1146-1150. https://doi.org/10.1126/science.284.5417.1146

Burns, J. A., Simonelli, D. P., Showalter, M. R., Hamilton, D. P., Porco, C. D., Throop, H., \& Esposito, L. W (2004). Jupiter's ring-moon system. In F. Bagenal, T. E. Dowling, \& W. B. McKinnon (Eds.), Jupiter. The Planet, Satellites and Magnetosphere (pp. 241-262), Cambridge, UK, Cambridge University Press, ISBN 0-521-81808-7, 2004.

Close, S., Linscott, I., Lee, N., Johnson, T., Strauss, D., Goel, A., et al. (2013). Detection of electromagnetic pulses produced by hypervelocity micro particle impact plasmas. Physics of Plasmas, 20(9), 092-102. https://doi.org/10.1063/1.4819777

Collette, A., Grün, E., Malaspina, D., \& Sternovsky, Z. (2014). Micrometeoroid impact charge yield for common spacecraft materials. J. Geophys. Res.: Space Physics, 119(8), 6019-6026. https://doi.org/10.1002/2014JA020042

Collette, A., Malaspina, D. M. \& Sternovsky, Z. (2016). Characteristic temperatures of hypervelocity dust impact plasmas. J. Geophys. Res.: Space Physics, 121(9), 8182-8187. https://doi.org/10.1002/2015JA022220 
Collette, A., Meyer, G., Malaspina, D., \& Sternovsky, Z. (2015). Laboratory investigation of antenna signals from dust impacts on spacecraft. J. Geophys. Res.: Space Physics, 120(7), 5298-5305. https://doi.org/10.1002/2015JA021198

Connerney, J. E. P., Benn, M., Bjarno, J. B., Denver, T., Espley, J., Jorgensen, P. S., et al. (2017). The Juno magnetic field investigation. Space Science Reviews, 213(1-4), 39-138. https://doi.org/10.1007/s11214-017-0334-Z

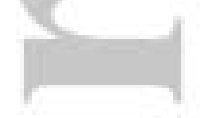

Connerney, J.E.P., Kotsiaros, S., Oliversen, R.J., et al. (2018). A new model of Jupiter's magnetic field from Juno's first nine orbits. Geophys Res. Lett., 45, doi:10.1002/2018GL077312.

de Pater, I., Showalter, M. R. \& Macintosh, B. (2008). Keck observations of the 2002-2003 Jovian ring plane crossing. Icarus, 195(1), 348-360.

https://doi.org/10.1016/j.icarus.2007.11.029

Fillius, R. W. (1976). The trapped radiation belts of Jupiter, in Jupiter, Ed. T. Gehrels, pp 896-927, University Arizona Press, Tucson.

Gurnett, D. A., Grün, E., Gallagher, D., Kurth, W. S., \& Scarf, F. L. (1983). Micron-sized particles detected near Saturn by the Voyager plasma wave instrument. Icarus, 53, 236-254.

Gurnett, D. A., Kurth, W. S., Granroth, L. J., Allendorf, S. C., \& Poynter, R. L. (1991). Micronsized particles detected near Neptune by the Voyager 2 plasma wave instrument. J. Geophys. Res., 96, 19,177. https://doi.org/10.1029/91JA01270 
Gurnett, D. A., Kurth, W. S., Scarf, F. L., Burns, J. A., Cuzzi, J. N., \& Grün, E. (1987). Micronsized particle impacts detected near Uranus by the Voyager 2 plasma wave instrument. $J$. Geophys. Res., 92, 14,959. https://doi.org/10.1029/JA092iA13p14959

Horányi, M., \& Juhász, A. (2010). Plasma conditions and the structure of the Jovian ring. J. Geophys. Res.: Space Physics, 115(A9). https://doi.org/10.1029/2010JA015472

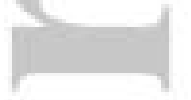

Kellogg, P. J., Goetz, K., \& Monson, S. J. (2016). Dust impact signals on the wind spacecraft. J. Geophys. Res.: Space Physics, 121(2), 966-991. https://doi.org/10.1002/2015JA021124

Kurth, W. S., Averkamp, T. F., Gurnett, D.A., \& Wang, Z. (2006). Cassini RPWS observations of dust in Saturn's E ring. Planet. Space Sci., 54, 988-998. https://doi.org/10.1016/j.pss.2006.05.011

Kurth, W. S., Hospodarsky, G. B., Kirchner, D. L., Mokrzycki, B. T., Averkamp, T. F., Robison, W. T., Piker, C. W., Sampl, M., \& Zarka, P. (2017). The Juno waves investigation. Space Science Reviews, 213(1-4), 347-392. doi:10.1007/s11214-017-0396-y

Lee, N., Close, S., Goel, A., Lauben, D., Linscott, I., Johnson, T., Strauss, D., Bugiel, S., Mocker, A., \& Srama, R. (2013). Theory and experiments characterizing hypervelocity impact plasmas on biased spacecraft materials. Physics of Plasmas, 20(3), 032901. https://doi.org/10.1063/1.4794331

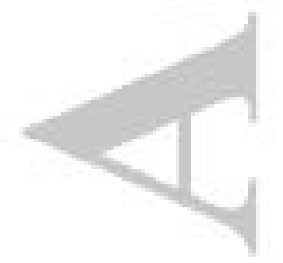


Lee, N., Close, S., Lauben, D., Linscott, I., Goel, A., Johnson, T., et al. (2012).

Measurements of freely-expanding plasma from hypervelocity impacts. International Journal of Impact Engineering, 44, 40-49. https://doi.org/10.1016/j.ijimpeng.2012.01.002

Malaspina, D.M., Horányi, M., Zaslavsky, A., Goetz, K., Wilson III, L.B., \& Kersten, K. (2014). Interplanetary and interstellar dust observed by the Wind/WAVES electric field instrument. Geophys. Res. Lett., 41(2), 266-272. https://doi.org/10.1002/2013GL058786

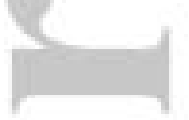

Meyer-Vernet, N. (1985). Comet Giacobini-Zinner diagnosis from radio measurements. Adv. Space Res., 5, 37-46. https://doi.org/10.1016/0273-1177(85)90065-1

Meyer-Vernet, N., Aubier, M. G., \& Pedersen, B. M. (1986). Voyager 2 at Uranus: Grain impacts in the ring plane. Geophys. Res. Lett., 13(7), 617-620.

https://doi.org/10.1029/GL013i007p00617

Meyer-Vernet, N., Lecacheux, A., Kaiser, M. L., \& Gurnett, D. A. (2009). Detecting nanoparticles at the radio frequencies: Jovian dust stream impacts on Cassini/RPWS. Geophys. Res. Lett., 36, 3103. https://doi.org/10.1029/2008GL036752

Meyer-Vernet, N., Lecacheux, A., \& Pedersen, B. M. (1996). Constraints on Saturn's E ring from the Voyager 1 radio astronomy instrument. Icarus, 123, 113-129.

https://doi.org/10.1006/icar.1996.0145

Meyer-Vernet, N., Moncuquet, M., Issautier, K., \& Lecacheux, A. (2014). The importance of monopole antennas for dust observations: Why Wind/WAVES does not detect nanodust. 
Geophys. Res. Lett., 41, 2716-2720. https://doi.org/10.1002/2014GL059988

Meyer-Vernet, N., Moncuquet, M., Issautier, K., \& Schippers, P. (2017). Frequency range of dust detection in space with radio and plasma wave receivers: Theory and application to interplanetary nanodust impacts on Cassini. J. Geophys. Res: Space Physics, 122(1), 8-22. https://doi.org/10.1002/2016JA023081

Nouzák, L., Hsu, S., Malaspina, D., Thayer, F. M., Ye, S.-Y., Pavlu, J., Nemecek, Z.,

Safrankova, J., \& Sternovsky, Z. (2018). Laboratory modeling of dust impact detection by the Cassini spacecraft. Planet. Space Sci., 156, 85-91. https://doi.org/10.1016/j.pss.2017.11.014

Nouzák, L., Sternovsky, Z., Horányi , M., Hsu, S., Pavlů , J., Shen, M.-H., \& Ye, S.-Y. (2019). Magnetic field effect on antenna signals induced by dust particle impacts. J. Geophys. Res: Space Physics. https://doi.org/10.1029/2019JA027245

Oberc, P. (1996). Electric antenna as a dust detector. Adv. Space Res., 17, 105-11. https://doi.org/10.1016/0273-1177(95)00766-8

Oberc, P., Parzydlo, W., \& Vaisberg, O. L. (1990). Correlations between the Vega 2 plasma wave (APV-N) and dust (SP-1) observations at Comet Halley. Icarus, 86, 314. https://doi.org/10.1016/0019-1035(90)90221-T

Ockert-Bell, M. E., Burns, J. A., Daubar, I. J., Thomas, P. C., Veverka, J., Belton, M. J. S., \& Klaasen, K. P. (1999). The structure of Jupiter's ring system as revealed by the Galileo imaging experiment. Icarus, 138(2), 188-213. https://doi.org/10.1006/icar.1998.6072 
Pantellini, F., Landi, S., Zaslavsky, A., \& Meyer-Vernet, N. (2012). On the unconstrained expansion of a spherical plasma cloud turning collisionless: Case of a cloud generated by a nanometre dust grain impact on an uncharged target in space. Plasma Phys. Controlled Fusion, 54(4), 045005, doi:10.1088/0741-3335/54/4/045005.

Pedersen, B. M., Meyer-Vernet, N., Aubier, M. G., \& Zarka, P. (1991). Dust distribution around Neptune: Grain impacts near the ring plane measured by the Voyager planetary radio astronomy experiment. J. Geophys. Res.: Space Physics, 96(S01), 19,187-19,196.

https://doi.org/10.1029/91JA01601

Schippers, P., Meyer-Vernet, N., Lecacheux, A., Belheouane, S., Moncuquet, M., Kurth, W. S., Mann, I., Mitchell, D. G., \& Andre, N. (2015). Nanodust detection between 1 and 5 AU using Cassini wave measurements. Astrophys. J., 806:77. doi:10.1088/0004-637X/806/1/77

Showalter, M. R., Burns, J. A., Cuzzi, J. N., \& Pollack, J. B. (1985). Discovery of Jupiter's 'gossamer' ring. Nature, 316, 526-528. https://doi.org/10.1038/316526a0

Showalter, M. R., Burns, J. A., Cuzzi, J. N., \& Pollack, J. B. (1987). Jupiter's ring system: New results on structure and particle properties. Icarus, 69(3), 458-498. https://doi.org/10.1016/0019-1035(87)90018-2

Showalter, M. R., de Pater, I., Verbanac, G., Hamilton, D. P., \& Burns, J. A. (2008). Properties and dynamics of Jupiter's gossamer rings from Galileo, Voyager, Hubble and Keck images. Icarus, 195, 361. https://doi.org/10.1016/j.icarus.2007.12.012 
Smith, B. A., Soderblom, L. A., Johnson, T. V., Ingersoll, A. P., Collins, S. A., Shoemaker, E. M., et al. (1979). The Jupiter System Through the Eyes of Voyager 1. Science, 204, 951972. https://doi.org/10.1126/science.204.4396.951

Tsintikidis, D., Gurnett, D. A., Granroth, L. J., Allendorf, S. C., \& Kurth, W. S. (1994). A revised analysis of micron-sized particles detected near Saturn by the Voyager 2 plasma wave instrument. J. Geophys. Res.: Space Physics, 99(A2), 2261-2270.

https://doi.org/10.1029/93JA02906

Tsurutani, B. T., Clay, D. R., Zhang, L. D., Dasgupta, B., Brinza, D., Henry, M., et al. (2004). Plasma clouds associated with Comet P/Borrelly dust impacts. Icarus, 167(1), 89-99. https://doi.org/10.1016/j.icarus.2003.08.021

Vaverka, J., Pellinen-Wannberg, A., Kero, J., Mann, I., De Spiegeleer, A., Hamrin, M., Norberg, C., \& Pitkänen, T. (2017). Detection of meteoroid hypervelocity impacts on the Cluster spacecraft: First results. J. Geophys. Res.: Space Physics, 122, 6485-6494. https://doi.org/10.1002/2016JA023755

Vaverka, J., Pavlu, J., Nouzák, L, Safrankova, J., Nemecek, Z., Mann, I., Ye, S.Y. \& Lindqvist, P. -A., (2019). One-year analysis of dust impact-like events onto the MMS spacecraft. Journal of Geophysical Research, 124, 8179-8190. https://doi.org/10.1029/2019JA027035

Wang, Z., Gurnett, D. A. Averkamp, T. F. Persoon, A. M., \& Kurth, W. S. (2006). Characteristics of dust particles detected near Saturn's ring plane. Planet. Space Sci., 54, 957- 
966. https://doi.org/10.1016/j.pss.2006.05.015

Ye, S.-Y., Gurnett, D.A., \& Kurth, W.S. (2016a). In-situ measurements of Saturn's dusty rings based on dust impact signals detected by Cassini RPWS. Icarus, 279, 51-61. https://doi.org/10.1016/j.icarus.2016.05.006

Ye, S.-Y., Gurnett, D. A., Kurth, W. S., Averkamp, T. F., Kempf, S., Hsu, H. W., Srama, R., \& Gruen, E. (2014b). Properties of dust particles near Saturn inferred from voltage pulses induced by dust impacts on Cassini spacecraft. J. Geophys. Res.: Space Physics, 119(8). doi:10.1002/2014ja020024

Ye, S.-Y., Gurnett, D. A., Kurth, W. S., Averkamp, T. F., Morooka, M., Sakai, S., \& Wahlund, J.-E., (2014a). Electron density inside Enceladus plume inferred from plasma oscillations excited by dust impacts. J. Geophys. Res.: Space Physics, 119(5), 3373-3380. https://doi.org/10.1002/2014JA019861

Ye, S.-Y., Kurth, W. S., Hospodarsky, G. B., Averkamp, T. F., \& Gurnett, D. A. (2016b). Dust detection in space using the monopole and dipole electric field antennas. J. Geophys. Res.: Space Physics, 121(12), 11,964-11,972. https://doi.org/10.1002/2016JA023266

Ye, S.-Y., Vaverka, J., Nouzák, L., Sternovsky, Z., Zaslavsky, A., Pavlu, J., Mann, I., Hsu, H.-W., Averkamp, T. F., Sulaiman, A. H., Pisa, D., Hospodarsky, G. B., Kurth, W. S., \& Horanyi, M. (2019). Understanding Cassini RPWS Antenna Signals Triggered by Dust Impacts. Geophys. Res. Lett., 46, 10,941-10,950. https://doi.org/10.1029/2019GL084150 
Ye, S.-Y., Averkamp, T.F., Kurth, W.S., Brennan, M, Bolton, S., Connerney, J.E.P., \& Joergensen, J.L. (2020). Juno Waves Detection of Dust Impacts near Jupiter (Version 1.0.0) [Data set]. Zenodo. http://doi.org/10.5281/zenodo.3706834

Zaslavsky, A. (2015). Floating potential perturbations due to micrometeoroid impacts:

Theory and application to S/WAVES data. J. Geophys. Res.: Space Physics, 120(2), 855-867. https://doi.org/10.1002/2014JA020635

Zaslavsky, A., Meyer-Vernet, N., Mann, I., Czechowski, A., Issautier, K., Le Chat, G., Pantellini, F., Goetz, K., Maksimovic, M., Bale, S. D., \& Kasper, J. C. (2012). Interplanetary dust detection by radio antennas: Mass calibration and fluxes measured by STEREO/WAVES. J. Geophys. Res., 117, A0510. https://doi.org/10.1029/2011JA017480 
Table 1. Lorentzian function fitting parameters for the dust impact rate vs. Z (distance from the ring plane) profiles of the first 21 perijoves. HWHM stands for half width at half maximum $(\mathrm{km}) . \mathrm{Z} 0$ is the offset of the peak impact rate from the ring plane $(\mathrm{km})$.

\begin{tabular}{|l|l|r|r|r|r|}
\hline perijove & UTC of peak impact rate & peak impact rate & radial distance & HWHM & Z0 \\
\hline PJ1 & 2016-240T12:52:12.770 & 6.1 & 75717.5 & 2611.7 & -137.5 \\
\hline PJ3 & 2016-346T17:05:45.608 & 4.8 & 75763.8 & 3360.8 & 244.2 \\
\hline PJ4 & 2017-033T12:59:35.849 & 4.4 & 75960.9 & 2949.2 & 235.5 \\
\hline PJ5 & $2017-086 T 08: 54: 48.138$ & 5.2 & 75150.5 & 3605 & -374.8 \\
\hline PJ6 & 2017-139T06:03:54.372 & 5.6 & 75262.6 & 2670.4 & 252.3 \\
\hline PJ7 & 2017-192T01:58:17.651 & 6.2 & 75362.7 & 1964.2 & -106.2 \\
\hline PJ8 & 2017-244T21:52:45.955 & 6 & 75434.9 & 2260.3 & -38.1 \\
\hline PJ9 & 2017-297T17:46:52.251 & 6.3 & 76068.7 & 2789.6 & -130.7 \\
\hline PJ10 & 2017-350T18:01:42.579 & 6.7 & 76446.7 & 2459.5 & -144.1 \\
\hline PJ11 & 2018-038T13:56:26.859 & 6.9 & 75696.8 & 2286.3 & 5.7 \\
\hline PJ12 & 2018-091T09:50:58.172 & 6.4 & 75787 & 2316.8 & 134.3 \\
\hline PJ13 & 2018-144T05:45:20.490 & 5.6 & 75850 & 2172.1 & 495.9 \\
\hline PJ14 & 2018-197T05:23:19.789 & 6.3 & 76023.5 & 3418.9 & 67.3 \\
\hline PJ15 & 2018-250T01:17:56.105 & 6 & 76125.5 & 3266.2 & 235.8 \\
\hline PJ16 & 2018-302T21:12:35.439 & 6 & 76251.5 & 3780.9 & 245 \\
\hline PJ17 & 2018-355T17:06:49.747 & 6 & 77921.2 & 2955.1 & 555.8 \\
\hline PJ18 & 2019-043T17:41:45.041 & 5.3 & 76517.9 & 3605.2 & 199.4 \\
\hline PJ19 & $2019-096 T 12: 22: 05.317$ & 6.5 & 78453.5 & 6083.2 & 507 \\
\hline PJ20 & $2019-149 T 08: 16: 37.656$ & 8.8 & 81558.4 & 2995.9 & 116.5 \\
\hline PJ21 & $2019-202 T 04: 11: 34.003$ & & &
\end{tabular}


Juno Waves Perijove 21, 21 July, Day 202, 2019

(a)

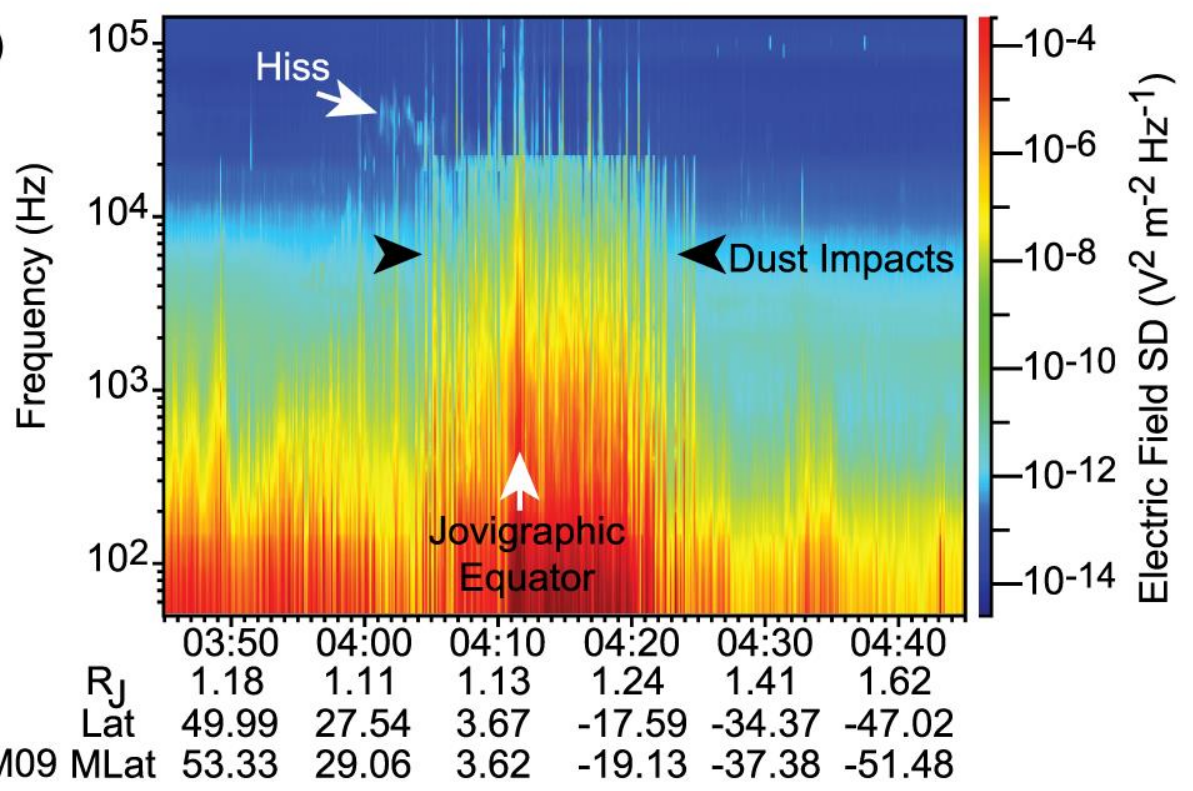

$\begin{array}{lllllll}\text { JRM09 MLat } 53.33 & 29.06 & 3.62 & -19.13 & -37.38 & -51.48\end{array}$
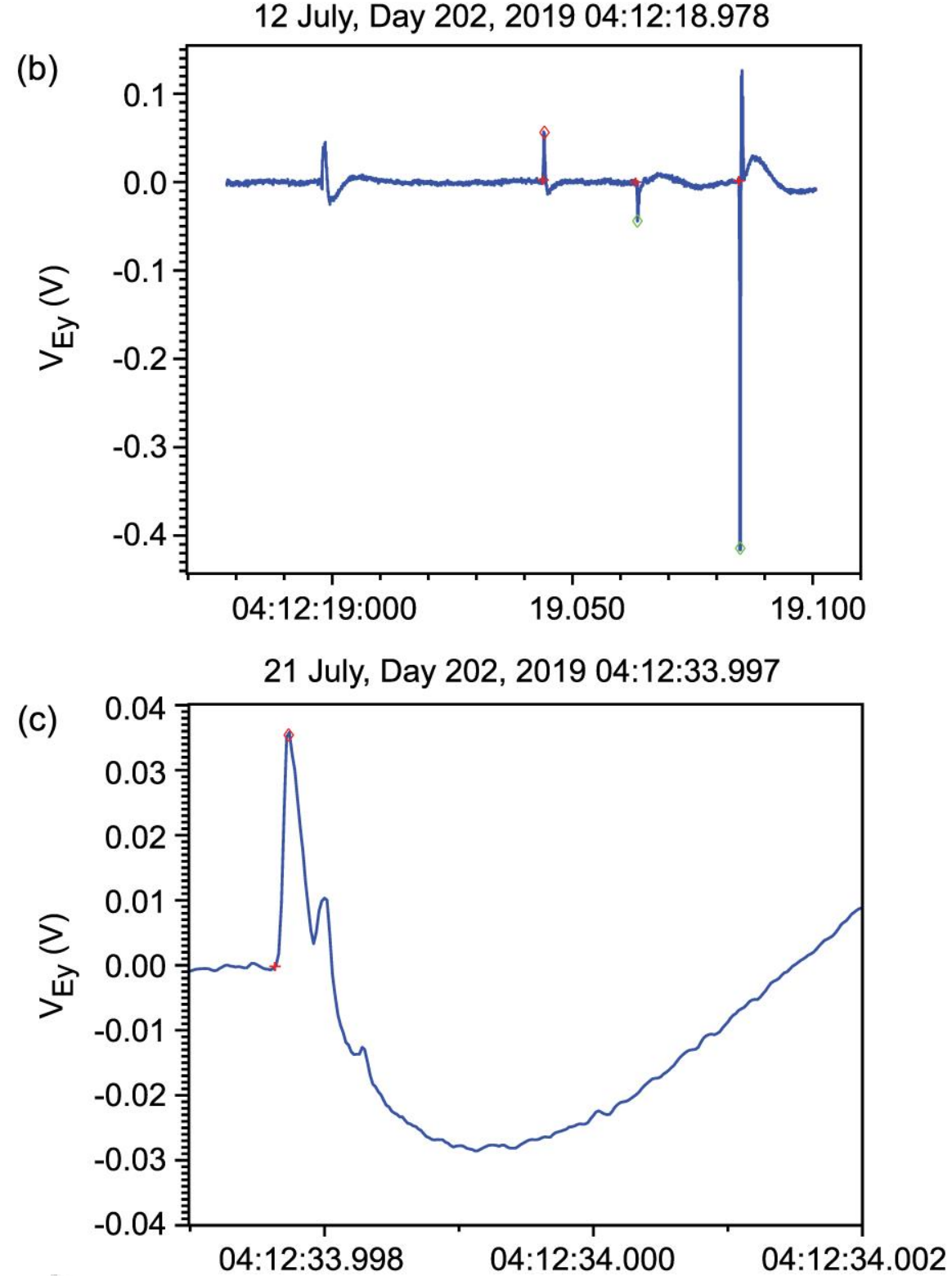

(C2020 American Geophysical Union. All rights reserved. 
Figure 1. (a) A Juno Waves spectrogram showing dust impact signals (broadband bursty structure shortly after 04:10) detected during PJ21 as a function of time, radial distance, latitude, and magnetic latitude computed using the JRM09 (Connerney et al., 2018) magnetic field model. (b) A waveform snapshot of Waves low frequency receiver burst mode showing individual impact signals. (c) An expanded view of a portion of the waveform snapshot showing the detailed structure of an impact signal. 


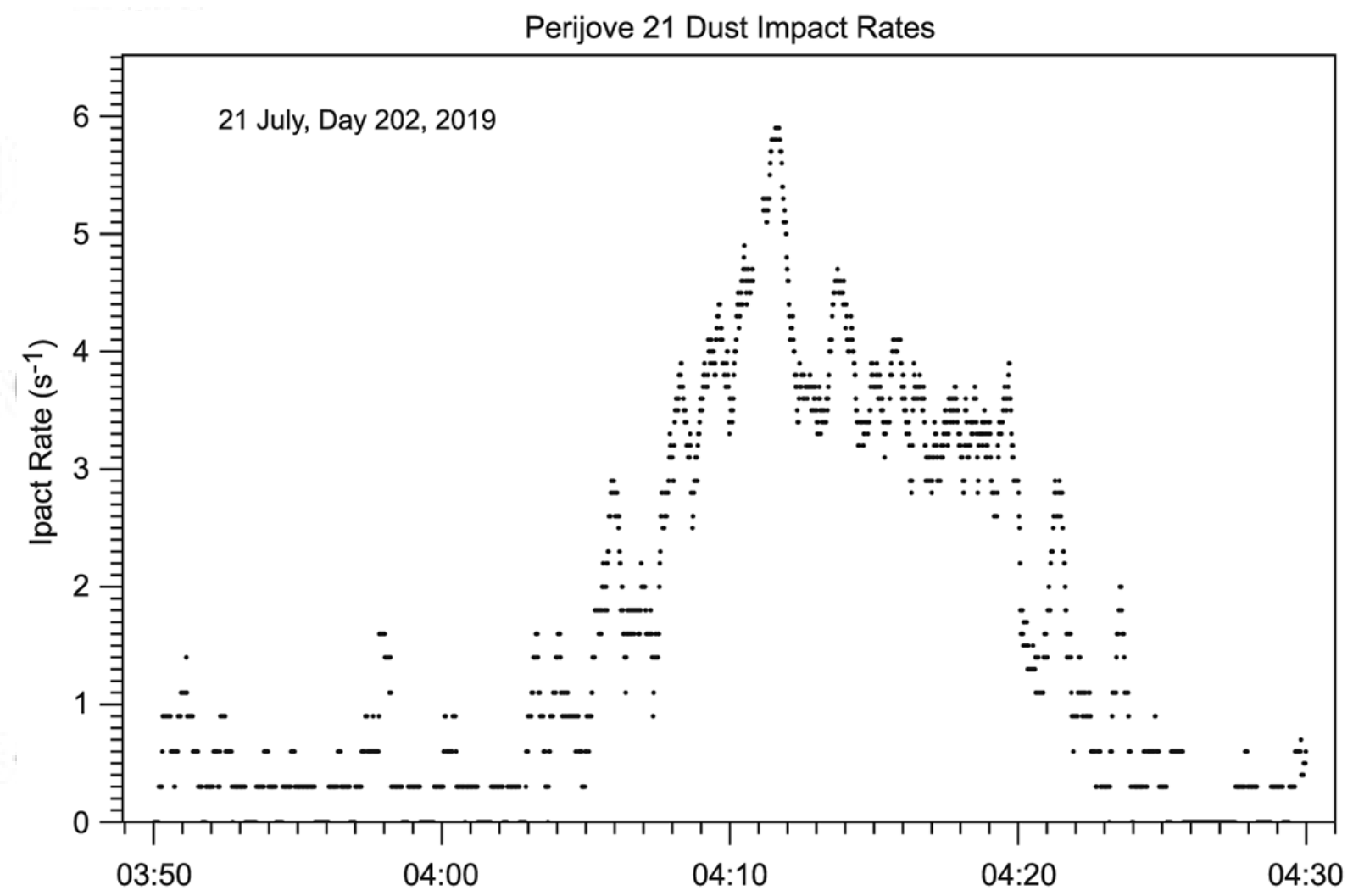

Figure 2. Impact rates vs. time recorded during perijove 21, smoothed using a 25 -second sliding average. The discrete nature of the rates most obvious at the lower rates results from the detection of an integer number of events within a 123-ms waveform capture, combined with the subsequent sliding average. 


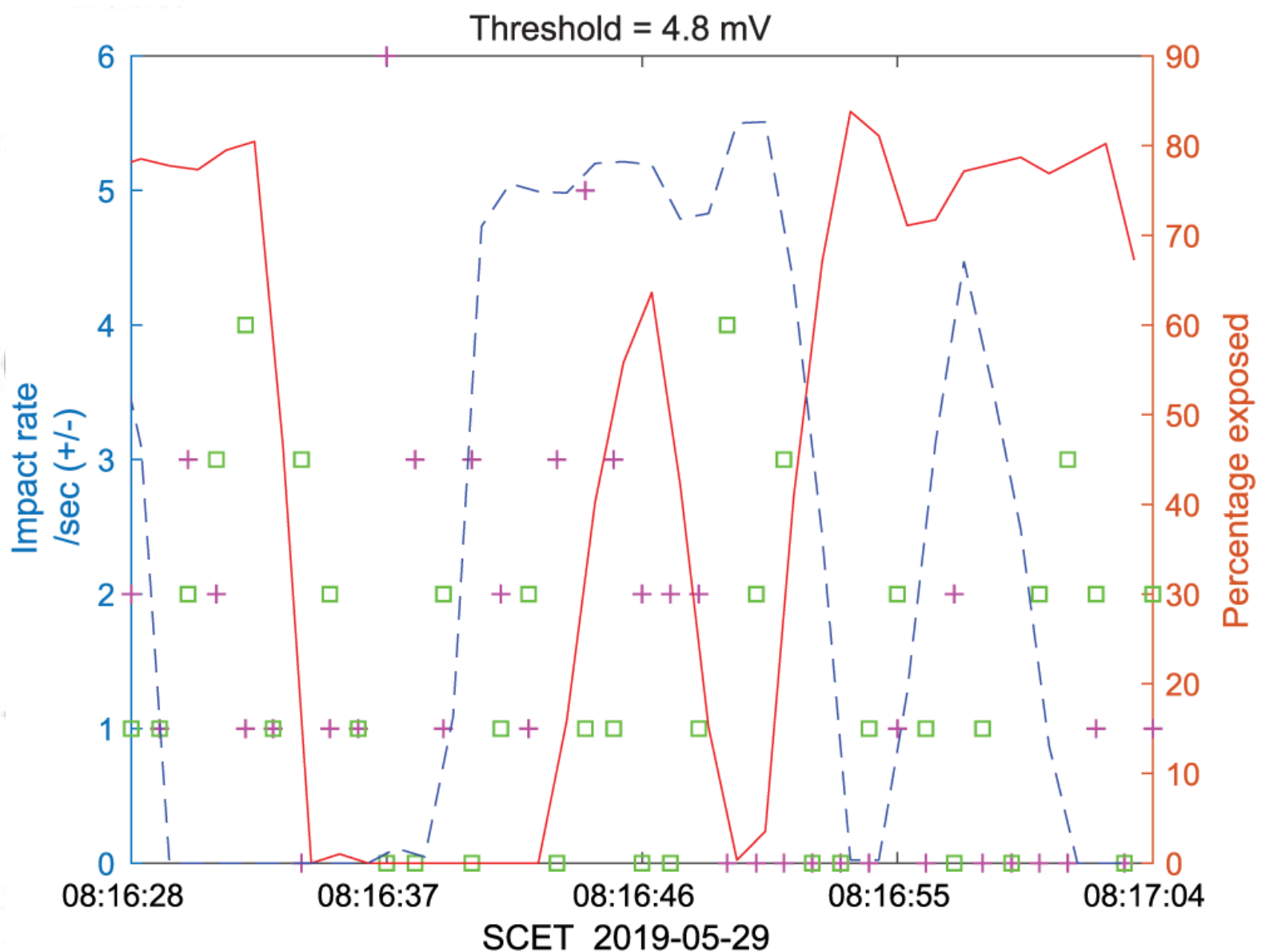

Figure 3. Comparison of impact rates (separated by polarity) with the percentage of antenna area exposed to ram direction. The red solid line indicates the percentage of positive $\mathrm{X}$ antenna exposed to impacts. The blue dashed line indicates the percentage of negative $\mathrm{X}$ antenna exposed to impacts. The pink plus sign and the green squares show the positive and negative impact rates detected during $\mathrm{PJ} 20$. 
(a)

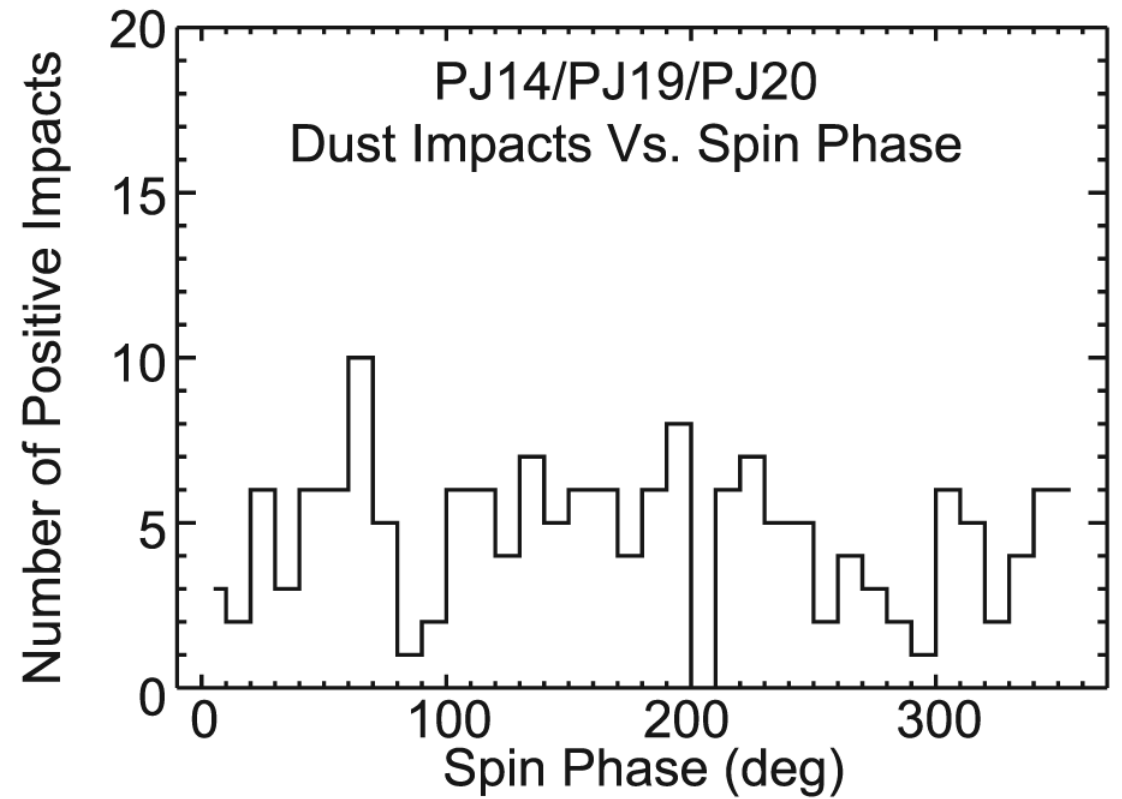

(b)

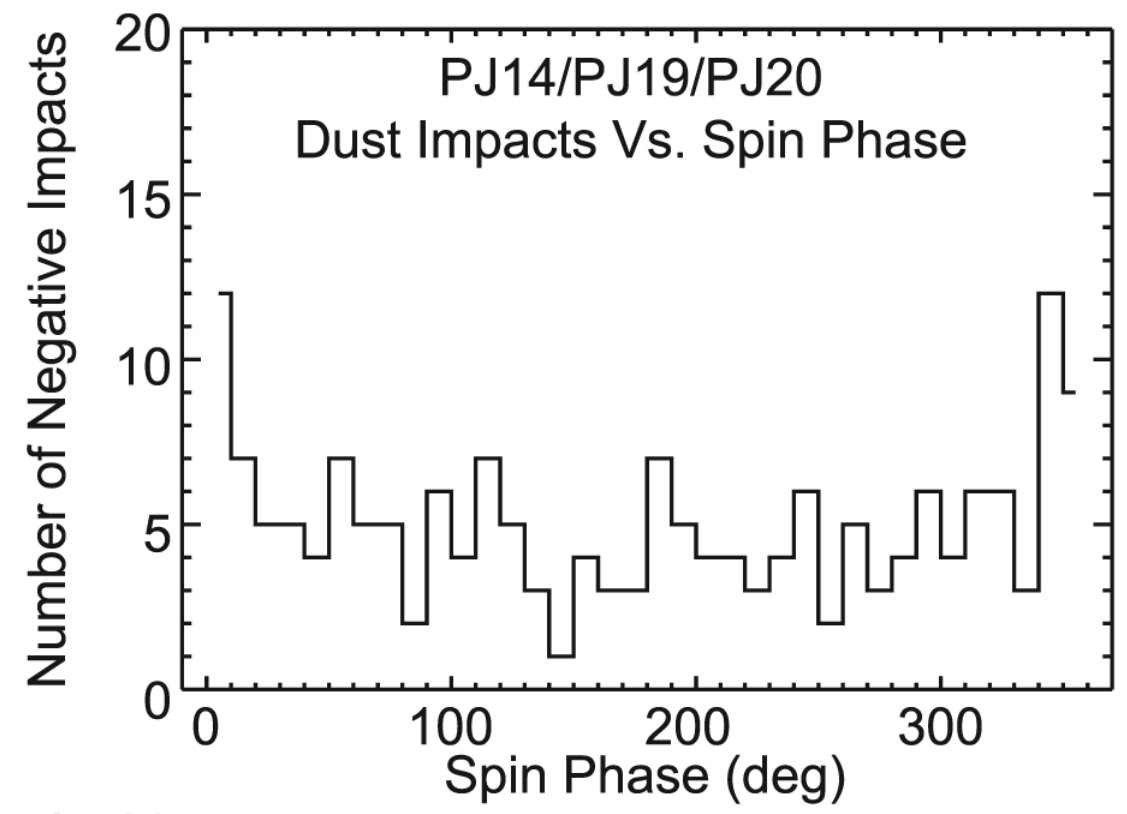

(c)

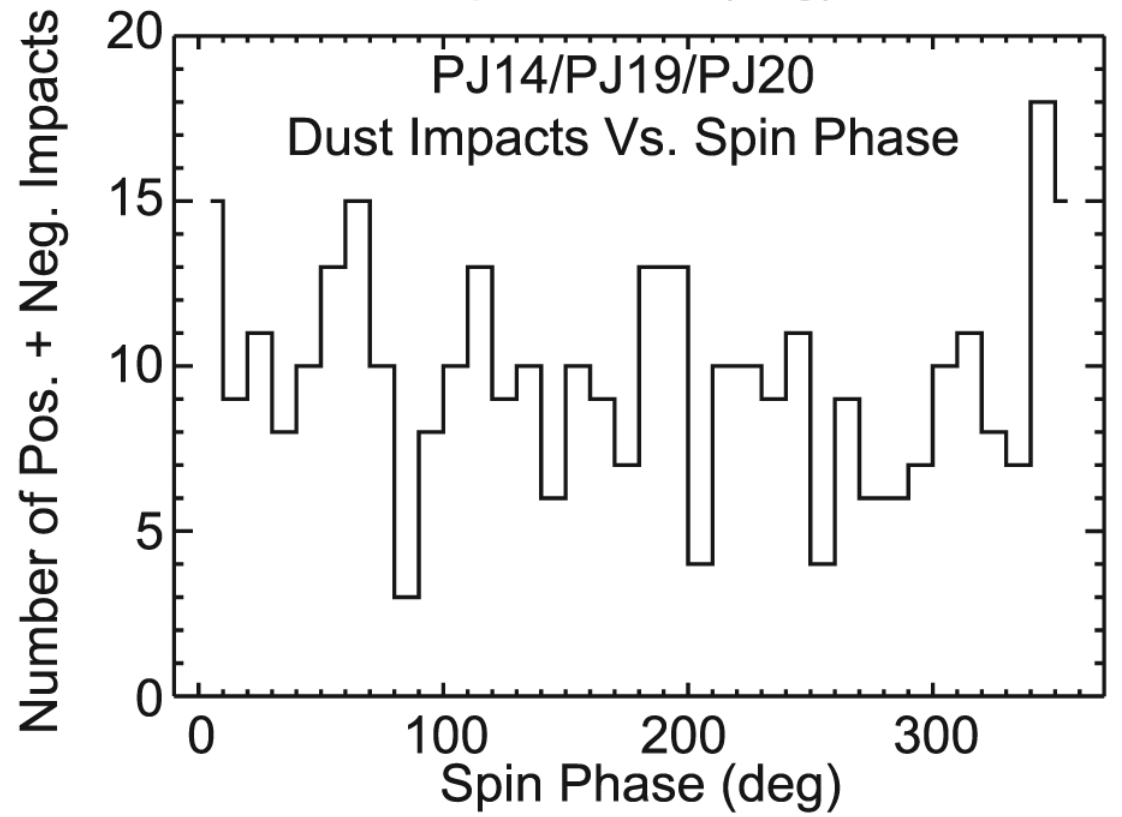

(C2020 American Geophysical Union. All rights reserved. 
Figure 4. Number of impacts detected as a function of an arbitrary rotation phase of the spacecraft: (a) positive events, (b) negative events, and (c) both polarities. The lack of significant variation with rotation phase suggests the impacts are not related to the orientation or exposure of the Waves electric antenna to the dust ram direction.

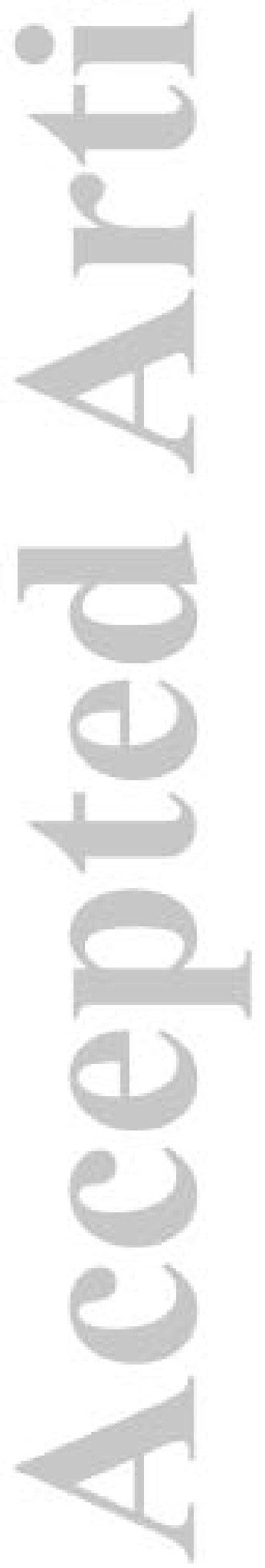




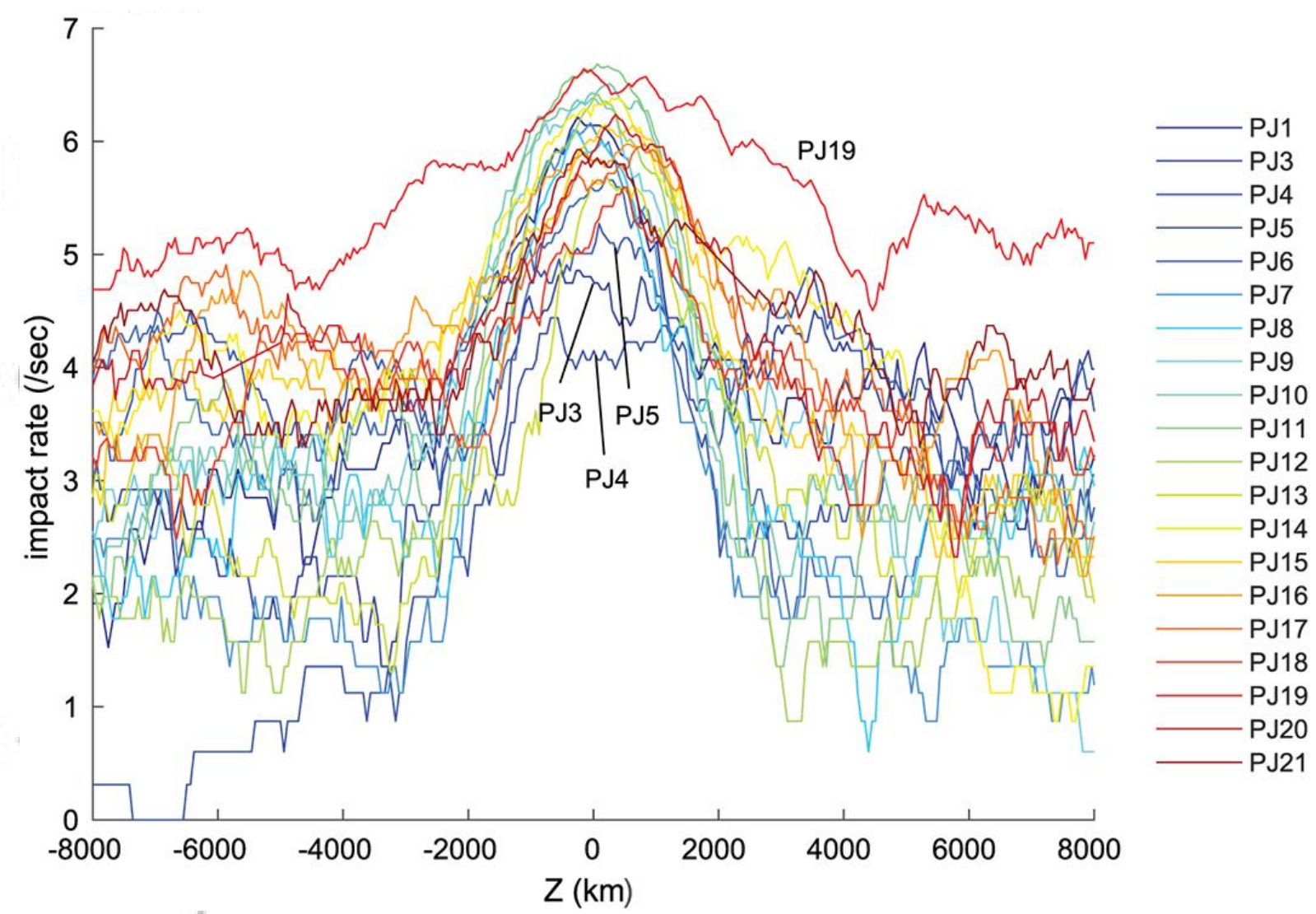

Figure 5. Dust impact rates detected by Waves as a function of distance from the Jovigraphic equator. Perijoves 1 and $3-21$ are plotted individually; during PJ2 no science data were collected. 


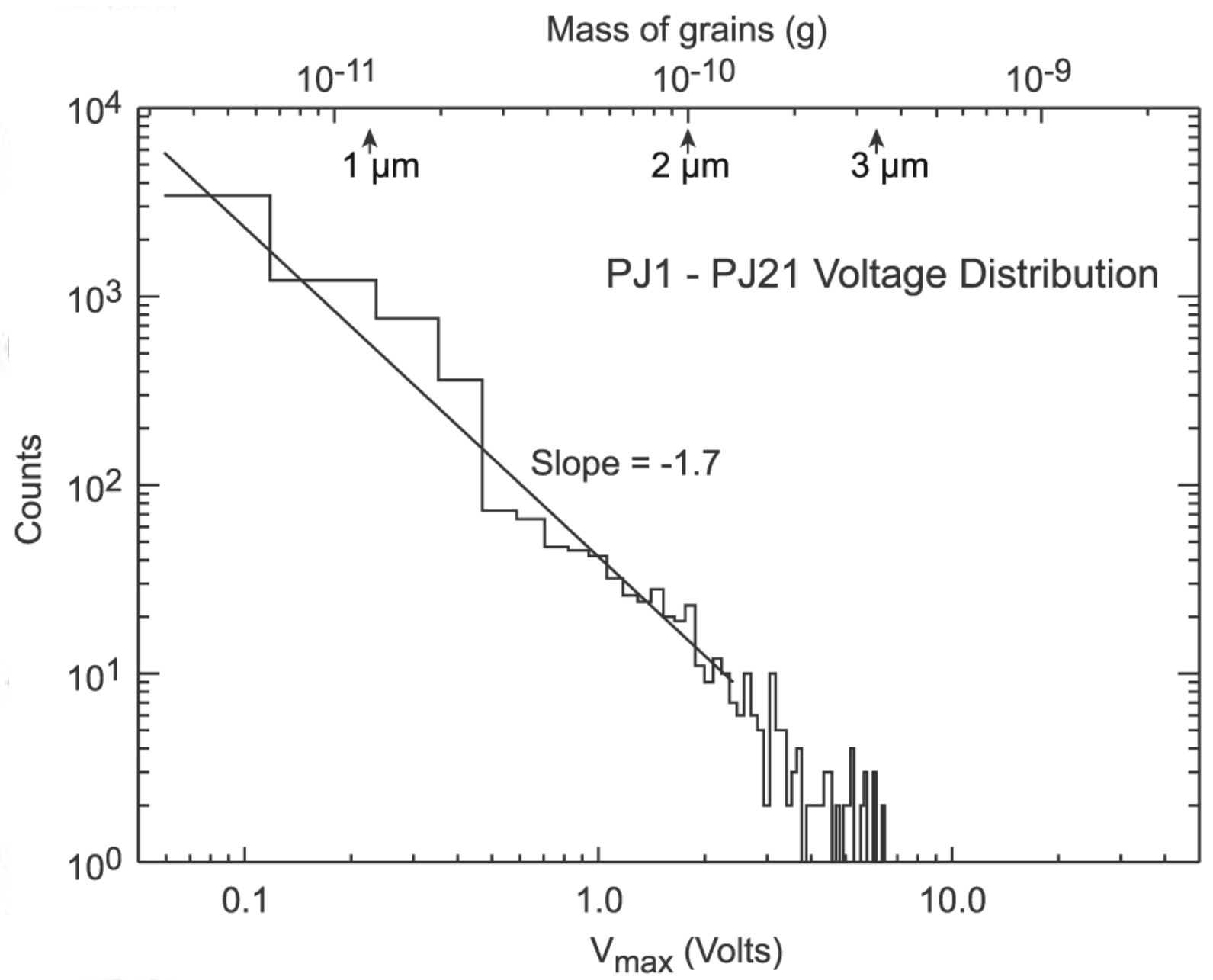

Figure 6. Voltage distribution of the dust impact signals detected by Waves during perijoves 1 - 21. The differential voltage (mass) distribution slope -1.7 , equivalent to a differential size distribution slope of -5.1. This analysis uses all detected impacts that were not clipped, accounting for the receiver attenuation in place at the time of detection. Since the voltage of the impulse is proportional to mass, and therefore, size, an approximate mass scale (top) is matched to the voltage axis. Grain sizes assuming a dust density of $3 \mathrm{~g} / \mathrm{cm}^{3}$ are shown at the top. See text. 


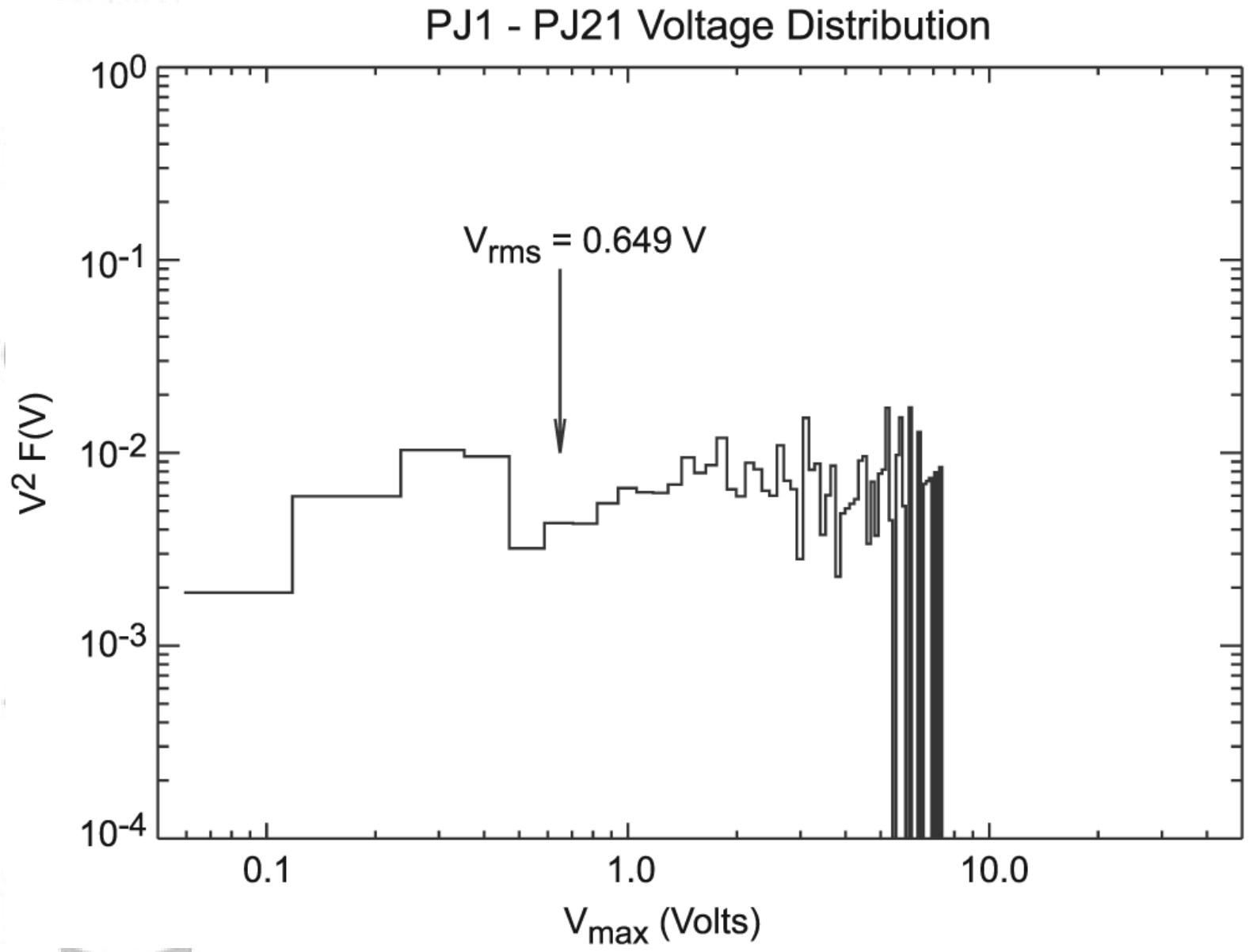

Figure 7. $\mathrm{V}^{2} \mathrm{~F}(V)$ distribution of dust impacts from perijoves 1 and 3 through 21. 

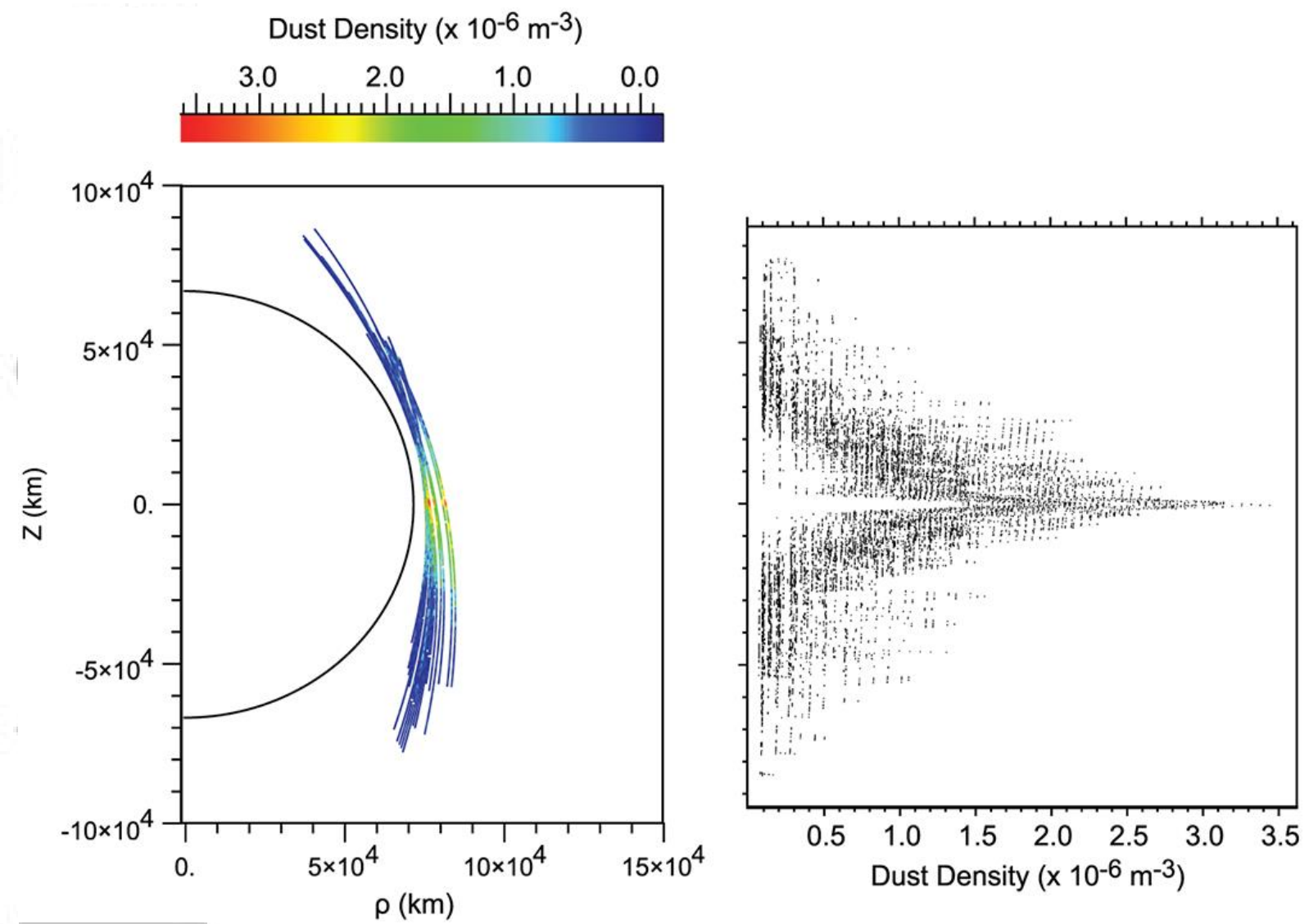

Figure 8. Density of detected dust grains near Jupiter from perijoves 1 and 3 through 21 . (left)

Dust density as a function of Z, distance from the equator and distance from Jupiter's rotation axis. (right) Scatter plot of dust density as a function of distance from the equator. 

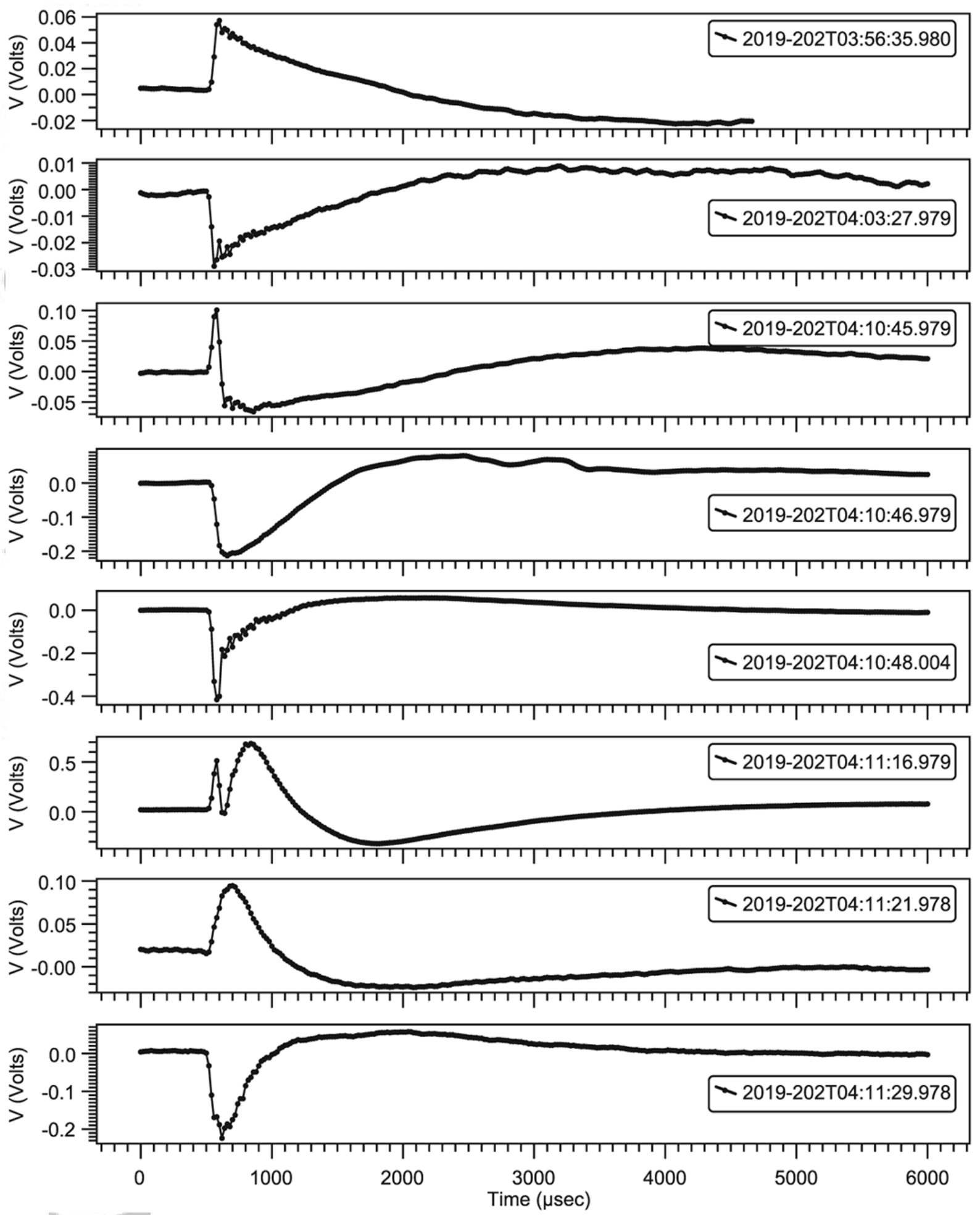

Figure 9. Sample waveforms of dust impact signals detected by Waves during PJ21. 

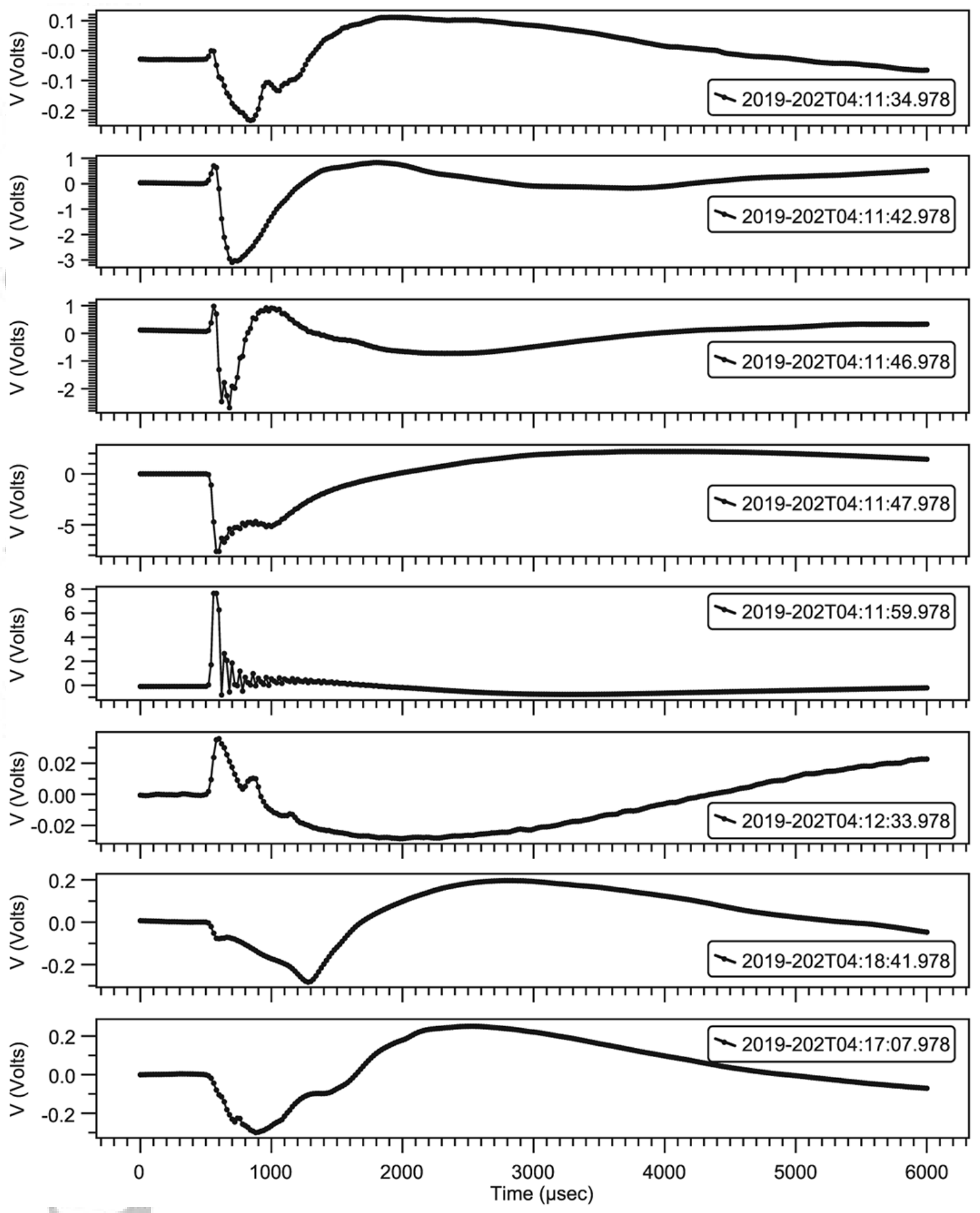

Figure 10. Sample waveforms of dust impact signals detected by Waves during PJ21. 